PROCEEDINGS OF THE

AMERICAN MATHEMATICAL SOCIETY

Volume 131, Number 10, Pages 2993-3002

S 0002-9939(02)06876-4

Article electronically published on December 30, 2002

\title{
HOPF ALGEBROIDS AND H-SEPARABLE EXTENSIONS
}

\author{
LARS KADISON \\ (Communicated by Martin Lorenz)
}

\begin{abstract}
Since an H-separable extension $A \mid B$ is of depth two, we associate to it dual bialgebroids $S:=$ End $_{B} A_{B}$ and $T:=\left(A \otimes_{B} A\right)^{B}$ over the centralizer $R$ as in Kadison-Szlachányi. We show that $S$ has an antipode $\tau$ and is a Hopf algebroid. $T^{\mathrm{op}}$ is also Hopf algebroid under the condition that the centralizer $R$ is an Azumaya algebra over the center $Z$ of $A$. For depth two extension $A \mid B$, we show that End ${ }_{A} A \otimes_{B} A \cong T \ltimes$ End $_{B} A$.
\end{abstract}

\section{INTRODUCTION AND PRELIMINARIES}

There is a notion introduced in 1968 by Hirata [7] of an H-separable extension of noncommutative rings, which has been studied intensively in connection with simple rings, skew group rings and skew polynomial rings by S. Ikehata, K. Sugano, G. Szeto and others. In many ways H-separable extension has a theory parallel to that of depth two subfactors in von Neumann algebra theory, the explanation emerging that both are special cases of depth two ring extensions [12, Kadison-Szlachányi].

Hopf algebroids over noncommutative rings were introduced by $\mathrm{Lu}[14$ in connection with quantization of Poisson groupoids in Poisson geometry. Examples of Hopf algebroids have also come from solutions to dynamical Yang-Baxter equations found by Etingof and Varchenko [5], although Etingof and Nikshych [4] showed that these are of a special self-dual type called weak Hopf algebras 1, Böhm-Szlachányi]. A bialgebroid $S$, i.e., a Hopf algebroid without antipode, and its $R$-dual $T$ has been associated with a depth two ring extension $A \mid B$ with centralizer $R$ in [12]. $S$ acts from the left on the over-ring $A$ such that the right endomorphism ring is isomorphic to a smash product $A \rtimes S$ [12]. Moreover, $T$ acts from the right on the left endomorphism ring $\mathcal{E}$ [12] such that the endomorphism ring End ${ }_{A} A \otimes_{B} A$ is similarly isomorphic to a smash product $T \ltimes \mathcal{E}$, as we show in this section.

In this paper we also show via the depth two theory in 12 that the bialgebroid $S$ of an H-separable extension $A$ over subring $B$ has Hopf algebroid structure over $R$. If additionally $R$ is Azumaya over the center of $A, T$ is a Hopf algebroid as well. We summarize the results of the papers of Nikshych, Vainerman, Szlachányi and the author [10, 11, 17, 12] together with this paper in the table below, from which we see the dual bialgebroids determined by a depth two extension $A \mid B$ converging

Received by the editors January 11, 2002 and, in revised form, April 22, 2002.

2000 Mathematics Subject Classification. Primary 13B02, 16H05, 16W30, 46L37, 81R15.

The author thanks Tomasz Brzezinski and U.W.S. for discussions and a hospitable visit to Swansea in the fall of 2001, as well as NORDAG in Bergen for partial support. 


\begin{tabular}{|l|l|l|}
\hline \hline Depth Two Extension & Centralizer & $\begin{array}{l}\text { Dual Quantum Algebras } \\
\text { with Galois Actions }\end{array}$ \\
\hline Unrestricted & Unrestricted & Bialgebroid \\
H-separable extension & Azumaya & Hopf algebroid \\
Frobenius extension & Separable & Weak Hopf algebra \\
Frobenius extension & Trivial & Hopf algebra \\
Biseparable Frobenius extension & Trivial & Semisimple Hopf algebra \\
\hline \hline
\end{tabular}

to semisimple Hopf algebras via Hopf algebroids and weak Hopf algebras as we place more restrictive conditions on the depth two extension and its centralizer.

Let $B$ be a unital subring of $A$, an associative noncommutative ring with unit. Recall that the ring extension $A \mid B$ is said to be of depth two if

$$
A \otimes_{B} A \oplus * \cong \bigoplus^{n} A
$$

as natural $B$ - $A$ and $A$-B-bimodules [12]. Equivalently, there are elements $\beta_{i} \in S:=$ End ${ }_{B} A_{B}, t_{i} \in T:=\left(A \otimes_{B} A\right)^{B}$ (called a left D2 quasibasis) such that $\left(a, a^{\prime} \in A\right)$

$$
a \otimes a^{\prime}=\sum_{i} t_{i} \beta_{i}(a) a^{\prime}
$$

and a right D2 quasibasis $\gamma_{j} \in S, u_{j} \in T$ such that

$$
a \otimes a^{\prime}=\sum_{j} a \gamma_{j}\left(a^{\prime}\right) u_{j} .
$$

We fix both D2 quasibases for our work below.

For example, an H-separable extension $A \mid B$ is of depth two since the condition above on the tensor-square holds even more strongly as natural $A$ - $A$-bimodules [7]. Another example is a finitely generated (f.g.) projective algebra $A$ over commutative ground ring $B$, since left or right D2 quasibases are easily constructed from a dual basis.

As another example, consider a Hopf-Galois extension $A \mid B$ with $n$-dimensional Hopf $k$-algebra $H$ [13]. Recall that $H$ acts from the left on $A$ with subalgebra of invariants $B$, induces a dual right coaction $A \rightarrow A \otimes_{k} H^{*}, a \mapsto a_{(0)} \otimes a_{(1)}$, and Galois isomorphism $\beta: A \otimes_{B} A \stackrel{\cong}{\longrightarrow} A \otimes_{k} H^{*}$ given by $\beta\left(a \otimes a^{\prime}\right)=a a_{(0)}^{\prime} \otimes a_{(1)}^{\prime}$, which is an $A$-B-bimodule, right $H^{*}$-comodule morphism. It follows that $A \otimes_{B} A \cong \bigoplus^{n} A$ as $A$ - $B$-bimodules; as $B$ - $A$-bimodules there is a similar isomorphism by making use of the alternative Galois isomorphism $\beta^{\prime}$ given by $\beta^{\prime}\left(a \otimes a^{\prime}\right)=a_{(0)} a^{\prime} \otimes a_{(1)}$.

The paper [12] uncovered a bialgebroid with action and smash product structure within the Jones construction above a depth two ring extension $A \mid B$. In more detail, if $R$ denotes the centralizer of $B$ in $A$, a left $R$-bialgebroid structure on $S$ is given by the composition ring structure on $S$ with source and target mappings corresponding to the left regular representation $\lambda: R \rightarrow S$ and right regular representation $\rho$ : $R^{\text {op }} \rightarrow S$, respectively. Since these commute $\left(\lambda(r) \rho\left(r^{\prime}\right)=\rho\left(r^{\prime}\right) \lambda(r)\right.$ for every $r, r^{\prime} \in R$ ), we may induce an $R$-bimodule structure on $S$ solely from the left by

$$
r \cdot \alpha \cdot r^{\prime}:=\lambda(r) \rho\left(r^{\prime}\right) \alpha=r \alpha(-) r^{\prime} .
$$

Now an $R$-coring ("co-ring" 21]) structure $(S, \Delta, \varepsilon)$ is given by

$$
\Delta(\alpha):=\sum_{i} \alpha\left(-t_{i}^{1}\right) t_{i}^{2} \otimes_{R} \beta_{i}
$$


for every $\alpha \in S$, denoting $t_{i}=t_{i}^{1} \otimes t_{i}^{2} \in T$ by suppressing a possible summation, and

$$
\varepsilon(\alpha)=\alpha(1)
$$

satisfying the additional axioms of a bialgebroid (cf. Section 3), such as multiplicativity of $\Delta$ and a condition that makes sense of this requirement. We have the equivalent formula for the coproduct [12] Thm. 4.1]:

$$
\Delta(\alpha):=\sum_{j} \gamma_{j} \otimes_{R} u_{j}^{1} \alpha\left(u_{j}^{2}-\right) .
$$

The left action of $S$ on $A$ given by evaluation, $\alpha \triangleright a=\alpha(a)$, has invariant subring (of elements $a \in A$ such that $\alpha \triangleright a=\varepsilon(\alpha) a$ ) equal precisely to $B$ if the natural module $A_{B}$ is balanced [12]. This action is measuring since $\alpha_{(1)}(a) \alpha_{(2)}\left(a^{\prime}\right)=\alpha\left(a a^{\prime}\right)$ by Eq. (1).

The smash product $A \rtimes S$, which is $A \otimes_{R} S$ as abelian groups with associative multiplication given by

$$
(a \rtimes \alpha)\left(a^{\prime} \rtimes \alpha^{\prime}\right)=a\left(\alpha_{(1)} \triangleright a^{\prime}\right) \rtimes \alpha_{(2)} \alpha^{\prime},
$$

is isomorphic as rings to End $A_{B}$ via $a \otimes_{R} \alpha \mapsto \lambda_{a} \alpha$ [12].

In general $T=\left(A \otimes_{B} A\right)^{B}$ has a unital ring structure induced from $T \cong$ End ${ }_{A}\left(A \otimes_{B} A\right)_{A}$ via $F \mapsto F(1 \otimes 1)$, which is given by

$$
t t^{\prime}=t^{\prime 1} t^{1} \otimes t^{2} t^{2}
$$

for each $t, t^{\prime} \in T$. There are obvious commuting homomorphisms of $R$ and $R^{\mathrm{op}}$ into $T$ given by $r \mapsto 1 \otimes r$ and $r^{\prime} \mapsto r^{\prime} \otimes 1$, respectively. From the right, these two "source" and "target" mappings induce the $R$ - $R$-bimodule structure ${ }_{R} T_{R}$ given by

$$
r \cdot t \cdot r^{\prime}=\left(t^{1} \otimes t^{2}\right)\left(r \otimes r^{\prime}\right)=r t r^{\prime},
$$

the ordinary bimodule structure on a tensor product.

There is a right $R$-bialgebroid structure on $T$ with coring structure $(T, \Delta, \varepsilon)$ given by the two equivalent formulas

$$
\begin{gathered}
\Delta(t)=\sum_{i} t_{i} \otimes_{R}\left(\beta_{i}\left(t^{1}\right) \otimes_{B} t^{2}\right)=\sum_{j}\left(t^{1} \otimes_{B} \gamma_{j}\left(t^{2}\right)\right) \otimes_{R} u_{j}, \\
\varepsilon(t)=t^{1} t^{2} .
\end{gathered}
$$

By [12, Thm. 5.2] $\Delta$ is multiplicative and the other axioms of a right bialgebroid are satisfied.

As an example of $S$ and $T$, consider the Hopf-Galois extension $A \mid B$ of $k$-algebras introduced above. Since $\beta$ is an $A$ - $B$-isomorphism, we may compute that $T \cong$ $R \otimes_{k} H^{*}$ via $\beta$, which induces a smash product structure on $R \otimes H^{*}$ relative to the Miyashita-Ulbrich action of $H^{*}$ on $R$ from the right (3] for definition of this action). The well-known isomorphism End $A_{B} \cong A \rtimes H$ via $a \rtimes h \mapsto \lambda(a)(h \triangleright \cdot)$ restricts to $S \cong R \rtimes H$, i.e., $S$ is a smash product of $R$ with $H$ via the restriction of the left action of $H$ to $R$. In both cases, the $R$-coring structures are the trivial ones induced from the coalgebras $H$ and $H^{*}$.

There is a right action of $T$ on $\mathcal{E}:=$ End $_{B} A$ given by $f \triangleleft t=t^{1} f\left(t^{2}-\right)$ for $f \in \mathcal{E}$. This is a measuring action by Eq. (11) since

$$
\left(f \triangleleft t_{(1)}\right) \circ\left(g \triangleleft t_{(2)}\right)=\sum_{i} t_{i}^{1} f\left(t_{i}^{2} \beta_{i}\left(t^{1}\right) g\left(t^{2}-\right)\right)=f g \triangleleft t .
$$


The subring of invariants in $\mathcal{E}$ is $\rho(A)$ [12. We next show that, in analogy with End $A_{B} \cong A \rtimes S$, the smash product ring $T \ltimes \mathcal{E}$ is isomorphic to End ${ }_{A} A \otimes_{B} A$ via $\Psi$ given by

$$
\Psi(t \otimes f)\left(a \otimes a^{\prime}\right)=a t^{1} \otimes_{B} t^{2} f\left(a^{\prime}\right) .
$$

Proposition 1.1. $\Psi$ is a ring isomorphism $T \ltimes \mathcal{E} \stackrel{\cong}{\longrightarrow}$ End ${ }_{A} A \otimes_{B} A$.

Proof. We let $\mu: A \otimes_{B} A \rightarrow A$ denote the multiplication mapping defined on simple tensors by $a \otimes a^{\prime} \mapsto a a^{\prime}$. Letting $F \in$ End ${ }_{A} A \otimes_{B} A$, define

$$
\Phi(F)=\sum_{i} t_{i} \otimes_{R} \mu\left(\beta_{i} \otimes A\right) F(1 \otimes-) .
$$

We check that $\Phi \circ \Psi=$ id: given $t \otimes f \in T \otimes_{R} \mathcal{E}$,

$$
\sum_{i} t_{i} \otimes_{R} \mu\left(\beta_{i} \otimes A\right)\left(t^{1} \otimes t^{2} f(-)\right)=\sum_{i} t_{i} \beta_{i}\left(t^{1}\right) t^{2} \otimes f=t \otimes f .
$$

Next, given $F \in$ End ${ }_{A} A \otimes{ }_{B} A$, let $F^{1}(a) \otimes F^{2}(a):=F(1 \otimes a)$ noting that $F\left(a \otimes a^{\prime}\right)=$ $a F^{1}\left(a^{\prime}\right) \otimes F^{2}\left(a^{\prime}\right)$. We check that $\Psi \Phi=$ id:

$$
\Psi \Phi(F)\left(a \otimes a^{\prime}\right)=\sum_{i} a t_{i}^{1} \otimes t_{i}^{2} \beta_{i}\left(F^{1}\left(a^{\prime}\right)\right) F^{2}\left(a^{\prime}\right)=a F^{1}\left(a^{\prime}\right) \otimes F^{2}\left(a^{\prime}\right)=F\left(a \otimes a^{\prime}\right) .
$$

Thus $\Psi$ is a bijective linear mapping.

Using Eq. (6), we check that $\Psi$ is a ring isomorphism:

$$
\begin{aligned}
\Psi\left((t \ltimes f)\left(t^{\prime} \ltimes f^{\prime}\right)\right)\left(a \otimes a^{\prime}\right) & =\Psi\left(t t^{\prime}(1) \ltimes\left(f \triangleleft t^{\prime}(2)\right) f^{\prime}\right)\left(a \otimes a^{\prime}\right) \\
& =\sum_{i} a t_{i}^{1} t^{1} \otimes t^{2} t_{i}^{2} \beta_{i}\left(t^{\prime 1}\right) f\left(t^{\prime 2} f^{\prime}\left(a^{\prime}\right)\right) \\
& =a t^{\prime 1} t^{1} \otimes t^{2} f\left(t^{\prime 2} f^{\prime}\left(a^{\prime}\right)\right) \\
& =\Psi(t \ltimes f) \Psi\left(t^{\prime} \ltimes f^{\prime}\right)\left(a \otimes a^{\prime}\right) . \quad \square
\end{aligned}
$$

Sweedler [21] has defined the left and right $R$-dual rings of an $R$-coring. In the case of a left $R$-bialgebroid $H$ with $H_{R}$ and ${ }_{R} H$ finitely generated projective, such as $(S, \lambda, \rho, \Delta, \varepsilon)$ above, the left and right Sweedler $R$-dual rings are extended to right bialgebroids $H^{*}$ and ${ }^{*} H$ in [12. For example, $H^{*}$ has a natural nondegenerate pairing with $H$ denoted by $\left\langle h^{*}, h\right\rangle \in R$ for $h^{*} \in H^{*}, h \in H$. Then the $R$-bimodule structure on $H^{*}$, multiplication, and comultiplication are given below, respectively, where $R \stackrel{s}{\rightarrow} H \stackrel{t}{\leftarrow} R^{\text {op }}$ denotes the commuting morphism set-up of the bialgebroid $H$ :

$$
\begin{aligned}
\left\langle r \cdot h^{*} \cdot r^{\prime}, h\right\rangle & :=r\left\langle h^{*}, h t\left(r^{\prime}\right)\right\rangle, \\
\left\langle h^{*} g^{*}, h\right\rangle & :=\left\langle g^{*},\left\langle h^{*}, h_{(1)}\right\rangle \cdot h_{(2)}\right\rangle, \\
\left\langle h^{*}, h h^{\prime}\right\rangle & :=\left\langle h^{*}{ }_{(1)} \cdot\left\langle h^{*}{ }_{(2)}, h^{\prime}\right\rangle, h\right\rangle .
\end{aligned}
$$

Of course, the unit of $H^{*}$ is $\varepsilon_{H}$ while the counit on $H^{*}$ is $\varepsilon\left(h^{*}\right)=\left\langle h^{*}, 1_{H}\right\rangle$. Eq. (10) is the formula for multiplication [21, 3.2(b)].

There are similar formulas for the right bialgebroid structure on the left $R$-dual ${ }^{*} H$; see [12, 2.6]. In the particular case of the left bialgebroid $S$ of a depth two ring extension, it turns out that $T$ is isomorphic as $R$-bialgebroids to both $R$-duals, $S^{*}$ and ${ }^{*} S$ via the nondegenerate pairings $\langle t, \alpha\rangle=t^{1} \alpha\left(t^{2}\right) \in R$ and $[\alpha, t]=\alpha\left(t^{1}\right) t^{2}$ $(\alpha \in S, t \in T)[12,5.3]$. 


\section{Frobenius D2 extensions}

This section is independent of the rest of the paper, although it explains the interest in certain constructions on $B \subseteq A$ we considered above. If $A \mid B$ is a Frobenius D2 extension, such as a depth two subfactor of finite index [12, 6 , the various objects and results above come together into a Jones tower as follows. In the Frobenius case we have $A \otimes_{B} A \cong$ End $A_{B}$ via $a \otimes a^{\prime} \mapsto \lambda_{a} \circ E \circ \lambda_{a^{\prime}}$ for some "Frobenius homomorphism" $E \in \operatorname{Hom}_{B-B}(A, B)$. Its inverse sends the identity into $\sum_{i} x_{i} \otimes y_{i}$ where $\left\{x_{i}\right\},\left\{y_{i}\right\}$ are "dual bases" for $E: A \rightarrow B[9$. (This may be more familiar in the case $B$ is a subring of the center of $A$.) If we denote $A_{1}:=A \otimes_{B} A$ with the induced "E-multiplication" from composition of endomorphisms, we see that $A_{1}$ has cyclic $A$-bimodule generator $e_{1}:=1 \otimes 1$, and the tower of rings induced by $\lambda: A \hookrightarrow$ End $A_{B} \cong A_{1}$,

$$
B \hookrightarrow A \hookrightarrow A_{1}
$$

is isomorphic to

$$
B \hookrightarrow A \hookrightarrow A \rtimes S
$$

where the latter mapping is $a \mapsto a \rtimes 1$, via the isomorphism $A \rtimes S \stackrel{\cong}{\longrightarrow} A_{1}$ given by $a \rtimes \alpha \mapsto \sum_{i} a \alpha\left(x_{i}\right) e_{1} y_{i}$.

It is a basic fact in Frobenius extension theory that the extension $A_{1} \mid A$ above is Frobenius as well - with canonical Frobenius homomorphism and dual bases $E_{M}:=\mu: a e_{1} a^{\prime} \mapsto a a^{\prime},\left\{x_{i} e_{1}\right\},\left\{e_{1} y_{i}\right\}$ (several sources, e.g. [18). If we now iterate the endomorphism ring construction above using the left, instead of right, endomorphism ring, we have an anti-isomorphism instead:

$$
A_{2}:=A_{1} e_{2} A_{1} \rightarrow \text { End }{ }_{A} A_{1}
$$

where $e_{2}=1 \otimes 1 \in A_{1} \otimes_{A} A_{1}$, via $z e_{2} w \mapsto \rho(w) E_{M} \rho(z)$ for $z, w \in A_{1}$, with inverse given by $f \mapsto \sum_{i} x_{i} e_{1} e_{2} f\left(e_{1} y_{i}\right)$. Similarly we have an anti-isomorphism inducing $A_{1}^{\text {op }} \cong \mathcal{E}$. Again we consider a tower of rings induced by the monomorphism $\lambda: A_{1} \hookrightarrow A_{2}, w \mapsto w^{1} e_{1} w^{2} 1_{A_{2}}=w^{1} e_{1} e_{2} e_{1} w^{2}:$

$$
A \hookrightarrow A_{1} \hookrightarrow A_{2}
$$

which is anti-isomorphic to the tower induced by the anti-monomorphism $w \mapsto$ $1_{T} \ltimes w$,

$$
A \hookrightarrow A_{1} \hookrightarrow T \ltimes A_{1}^{\mathrm{op}}
$$

via the anti-isomorphism $T \ltimes A_{1}^{\text {op }} \cong A_{2}$ given by $t \ltimes w \mapsto w^{1} e_{1} e_{2} t^{1} e_{1} t^{2} w^{2}$ where $w=w^{1} e_{1} w^{2} \in A_{1}$ and $t \in T$.

Since $A \mid B$ is D2 Frobenius, we note that

$$
(A \rtimes S)^{\mathrm{op}} \cong \text { End }_{B} A \cong A_{1}^{\mathrm{op}}
$$

and End ${ }_{A} A \otimes_{B} A \cong\left(\operatorname{End}\left(A_{1}\right)_{A}\right)^{\text {op }}$. Hence recalling $T \cong S^{*} \cong{ }^{*} S$ and Proposition 1.1, we arrive at a Blattner-Montgomery-Takesaki duality result (like that in [15] for weak Hopf algebras):

$$
T \ltimes(A \rtimes S)^{\mathrm{op}} \cong\left(\operatorname{End}(A \rtimes S)_{A}\right)^{\mathrm{op}} .
$$




\section{Hopf Algebroids AND LU'S EXAMPLES}

With an adaptation to algebras over commutative ground rings, Lu's Hopf algebroid and examples are the following. Let $K$ be a commutative ring. We first review the definition of a left bialgebroid $(H, R, \tilde{s}, \tilde{t}, \Delta, \varepsilon)$, and next review the definition of antipode $\tau$ for $H$, which are the two components of a Hopf algebroid. $H$ and $R$ are $K$-algebras and all maps are $K$-linear. First, recall from 14 (and compare [2, 22] that the source and target maps $\tilde{s}$ and $\tilde{t}$ are algebra homomorphism and anti-homomorphism, respectively, of $R$ into $H$ such that $\tilde{s}(r) \tilde{t}\left(r^{\prime}\right)=\tilde{t}\left(r^{\prime}\right) \tilde{s}(r)$ for all $r, r^{\prime} \in R$. This induces an $R$ - $R$-bimodule structure on $H$ (from the left in this case) by $r \cdot h \cdot r^{\prime}=\tilde{s}(r) \tilde{t}\left(r^{\prime}\right) h(h \in H)$. With respect to this bimodule structure, $(H, \Delta, \varepsilon)$ is an $R$-coring (cf. 21]), i.e. with coassociative coproduct and $R$ - $R$-bimodule map $\Delta: H \rightarrow H \otimes_{R} H$ and counit $\varepsilon: H \rightarrow R$ (also an $R$-bimodule mapping). The image of $\Delta$, written in Sweedler notation, is required to satisfy

$$
a_{(1)} \tilde{t}(r) \otimes a_{(2)}=a_{(1)} \otimes a_{(2)} \tilde{s}(r)
$$

for all $a \in H, r \in R$. It then makes sense to require that $\Delta$ be homomorphic:

$$
\Delta(a b)=\Delta(a) \Delta(b), \quad \Delta(1)=1 \otimes 1
$$

for all $a, b \in H$. The counit must satisfy the following modified augmentation law:

$$
\varepsilon(a b)=\varepsilon(a s(\varepsilon(b)))=\varepsilon(a t(\varepsilon(b))), \quad \varepsilon\left(1_{H}\right)=1_{R} .
$$

The axioms of a right bialgebroid $H^{\prime}$ are opposite those of a left bialgebroid in the sense that $H^{\prime}$ obtains its $R$-bimodule structure from the right via its source and target maps and, from the left bialgebroid $H$ above, we have that $\left(H^{\mathrm{op}}, R, \tilde{t}^{\mathrm{op}}, \tilde{s}^{\mathrm{op}}, \Delta, \varepsilon\right)$ (in that precise order) is a right bialgebroid. For the explicit axioms, see 12, Section 2].

In addition, the left $R$-bialgebroid $H$ is a $H o p f$ algebroid $(H, R, \tau)$ if (antipode) $\tau: H \rightarrow H$ is an algebra anti-automorphism such that

(1) $\tau \tilde{t}=\tilde{s}$

(2) $\tau\left(a_{(1)}\right) a_{(2)}=\tilde{t}(\varepsilon(\tau(a)))$ for every $a \in A$;

(3) there is a linear section $\eta: H \otimes_{R} H \rightarrow H \otimes_{K} H$ to the natural projection $H \otimes_{K} H \rightarrow H \otimes_{R} H$ such that

$$
\mu(H \otimes \tau) \eta \Delta=\tilde{s} \varepsilon .
$$

For example, one may compute that if $\left(H^{\prime}, R^{\prime}, \tau^{\prime}\right)$ is another Hopf algebroid, then $\left(H \otimes H^{\prime}, R \otimes R^{\prime}, \tau \otimes \tau^{\prime}\right)$ is (the tensor) Hopf algebroid.

Lu's examples of bialgebroids and Hopf algebroids are the following. Given an algebra $C$ over commutative ground ring $K$ such that $C$ is finitely generated projective as a $K$-module, the following two are left bialgebroids over $C$ (with $\left.\otimes=\otimes_{K}\right)$ :

Example 3.1. The endomorphism algebra $E:=\operatorname{End}_{K} C$ with $\tilde{s}(c)=\lambda(c), \tilde{t}\left(c^{\prime}\right)=$ $\rho\left(c^{\prime}\right)$, coproduct $\Delta(f)\left(c \otimes c^{\prime}\right)=f\left(c c^{\prime}\right)$ for $f \in \operatorname{End}_{K} C$ after noting that $E \otimes_{C} E \cong$ $\operatorname{Hom}_{K}(C \otimes C, C)$ via $f \otimes g \mapsto\left(c \otimes c^{\prime} \mapsto f(c) g\left(c^{\prime}\right)\right)$. The counit is given by $\varepsilon(f)=f(1)$. We see that this is the left bialgebroid $S$ above when $B=K$, a subring in the center of $A$.

Example 3.2. The ordinary tensor algebra $C \otimes C^{\text {op }}$ with $\tilde{s}(c)=c \otimes 1, \tilde{t}\left(c^{\prime}\right)=1 \otimes c^{\prime}$ with bimodule structure $c \cdot c^{\prime} \otimes c^{\prime \prime} \cdot c^{\prime \prime \prime}=c c^{\prime} \otimes c^{\prime \prime} c^{\prime \prime \prime}$. Coproduct $\Delta\left(c \otimes c^{\prime}\right)=c \otimes 1 \otimes c^{\prime}$ after a simple identification, with counit $\varepsilon\left(c \otimes c^{\prime}\right)=c c^{\prime}$ for $c, c^{\prime} \in C$. $C \otimes C^{\text {op }}$ 
is a left $C$-bialgebroid by arguing as in [14], or [12, $N=K]$ since $C \mid K$ is D2. In addition, $\tau: C \otimes C^{\mathrm{op}} \rightarrow C \otimes C^{\mathrm{op}}$, defined as the twist $\tau\left(c \otimes c^{\prime}\right)=c^{\prime} \otimes c$, is an antipode satisfying the axioms of a Hopf algebroid (in addition, $\tau^{2}=$ id, an involutive antipode).

A bialgebroid homomorphism from $\left(H_{1}, R_{1}, s_{1}, t_{1}, \Delta_{1}, \varepsilon_{1}\right)$ into $\left(H_{2}, R_{2}, s_{2}, t_{2}, \Delta_{2}\right.$, $\left.\varepsilon_{2}\right)$ consists of a pair of algebra homomorphisms, $F: H_{1} \rightarrow H_{2}$ and $f: R_{1} \rightarrow R_{2}$, such that four squares commute: $F s_{1}=s_{2} f, F t_{1}=t_{2} f, \Delta_{2} F=p(F \otimes F) \Delta_{1}$ and $\varepsilon_{2} F=f \varepsilon_{1}$, where $f$ induces an $R_{1}-R_{1}$-bimodule structure on $H_{2}$ via "restriction of scalars," $p: H_{2} \otimes_{R_{1}} H_{2} \rightarrow H_{2} \otimes_{R_{2}} H_{2}$ is the canonical mapping and $F: R_{1} H_{1 R_{1}} \rightarrow$ $R_{1} H_{2 R_{1}}$ is a bimodule homomorphism since

$$
F\left(r \cdot h \cdot r^{\prime}\right)=F\left(s_{1}(r) t_{1}\left(r^{\prime}\right) h\right)=s_{2}(f(r)) t_{2}\left(f\left(r^{\prime}\right)\right) F(h)=r \cdot_{f} F(h) \cdot f r^{\prime} .
$$

Proposition 3.3. If $F: H_{1} \rightarrow H_{2}$ and $f: R_{1} \rightarrow R_{2}$ are ring isomorphisms and $\tau_{1}$ is an antipode for $H_{1}$, then $\tau_{2}:=F \tau_{1} F^{-1}$ is an antipode for $H_{2}$.

The proof is an easy checking for $\tau_{2}$ of the axioms above and therefore omitted. As an example of a bialgebroid homomorphism with fixed base ring, let $C$ be the algebra introduced above and $\tilde{F}: C \otimes C^{\mathrm{op}} \rightarrow \operatorname{End}_{K} C$ be defined by $\tilde{F}\left(c \otimes c^{\prime}\right)\left(c^{\prime \prime}\right)=$ $c c^{\prime \prime} c^{\prime}$. The following is a consequence of the well-known Azumaya theorem (cf. [14] $3.8]$ and [9, 5.9]).

Proposition 3.4. $\tilde{F}: C \otimes_{K} C^{\mathrm{op}} \rightarrow \operatorname{End}_{K} C$ is a bialgebroid isomorphism if $C$ is an Azumaya K-algebra.

\section{H-SEPARABLE EXTENSIONS}

Again let $B$ be a subring of $A$ with centralizer subring $R$, endomorphism ring $S=$ End ${ }_{B} A_{B}$ and $\operatorname{ring} T=\left(A \otimes_{B} A\right)^{B}$. The definition and proposition below are due to [7, 8, Hirata].

Lemma \& Definition 4.1. $A \mid B$ is $H$-separable if $A \otimes_{B} A \oplus * \cong \bigoplus^{n} A$ as $A$ - $A$ bimodules. Equivalently, $A \mid B$ is $H$-separable if there are elements $e_{i} \in\left(A \otimes_{B} A\right)^{A}$ and $r_{i} \in R$ (a so-called H-separability system) such that

$$
1 \otimes 1=\sum_{i} r_{i} e_{i}
$$

For example, an Azumaya algebra $A \mid Z$ is $\mathrm{H}$-separable [7. We note that $e_{i} \in T$, and for $a, a^{\prime} \in A$,

$$
a \otimes a^{\prime}=\sum_{i} e_{i} \rho_{r_{i}}(a) a^{\prime}=\sum_{i} a \lambda_{r_{i}}\left(a^{\prime}\right) e_{i}
$$

whence $e_{i}, \lambda_{r_{i}}$ is a right D2 quasibasis and $e_{i}, \rho_{r_{i}}$ is a left D2 quasibasis for $A \mid B$.

We next let $Z$ denote the center of $A$.

Proposition 4.2. If $A \mid B$ is an $H$-separable extension, then

(1) $R$ is an f.g. projective $Z$-module;

(2) $R \otimes_{Z} R^{\text {op }} \cong S$ via $\phi: r \otimes r^{\prime} \mapsto \lambda_{r} \rho_{r^{\prime}}$;

(3) $T^{\mathrm{op}} \cong \operatorname{End}_{Z} R$ via $t \mapsto \lambda\left(t^{1}\right) \rho\left(t^{2}\right)$.

Proof. We offer some short alternative proofs to these facts. $R_{Z}$ is f.g. projective since for each $r \in R$, we note that $r=r_{i} e_{i}^{1} r e_{i}^{2}$ where summation over $i$ is understood and for each $i, r \mapsto e_{i}^{1} r e_{i}^{2}$ defines a map in $\operatorname{Hom}_{Z}(R, Z)$. 
The inverse $S \rightarrow R \otimes_{Z} R^{\text {op }}$ to the map $\phi$ above is given by $\alpha \mapsto \alpha\left(e_{i}^{1}\right) e_{i}^{2} \otimes r_{i}$, since $\alpha\left(e_{i}^{1}\right) e_{i}^{2} a r_{i}=\alpha\left(a e_{i}^{1}\right) e_{i}^{2} r_{i}=\alpha(a)(a \in A)$, while $r e_{i}^{1} r^{\prime} e_{i}^{2} \otimes_{Z} r_{i}=r \otimes e_{i}^{1} r^{\prime} e_{i}^{2} r_{i}=r \otimes r^{\prime}$ for $r, r^{\prime} \in R$.

The inverse $\operatorname{End}_{Z} R \rightarrow T^{\mathrm{op}}$ to the second mapping above is given by $g \mapsto g\left(r_{i}\right) e_{i}$, since for each $t=t^{1} \otimes t^{2} \in T, t^{1} r_{i} t^{2} e_{i}=t^{1} r_{i} e_{i} t^{2}=t$, while for each $r \in R$ $g \in \operatorname{End}_{Z} R, g\left(r_{i}\right) e_{i}^{1} r e_{i}^{2}=g\left(r_{i} e_{i}^{1} r e_{i}^{2}\right)=g(r)$.

\section{When $S$ And $T$ ARe Hopf Algebroids}

Putting together Proposition 4.2(2) with Example 3.2 and the fact from Section 1 that $S=$ End ${ }_{B} A_{B}$ is a left bialgebroid, we are led to the following.

Theorem 5.1. If $A \mid B$ is H-separable, then the isomorphism $\phi: R \otimes_{Z} R^{\mathrm{op}} \rightarrow S$ given in Proposition 4.2(2) is an isomorphism of bialgebroids; whence $S$ is a Hopf algebroid.

Proof. It suffices by Proposition 3.3 to check the commutativity of four diagrams in the definition of bialgebroid homomorphism

$$
\phi:\left(R \otimes R^{\mathrm{op}}, R, \tilde{s}, \tilde{t}, \Delta, \varepsilon\right) \rightarrow\left(S, R, \lambda, \rho, \Delta^{\prime}, \varepsilon^{\prime}\right) .
$$

First, given $r \in R, \phi(\tilde{s}(r))=\phi(r \otimes 1)=\lambda(r)$, and $\phi(\tilde{t}(r))=\phi(1 \otimes r)=\rho(r)$.

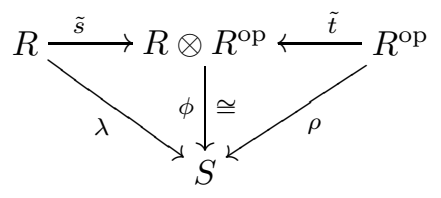

Second, for $r, r^{\prime} \in R$ we have $\varepsilon^{\prime} \phi\left(r \otimes r^{\prime}\right)=r r^{\prime}=\varepsilon\left(r \otimes r^{\prime}\right)$. Finally, we use the left D2 quasibasis $t_{i} \in T, \beta_{i} \in S$ with Eq. (2), and then a canonical isomorphism to compute

$$
\begin{aligned}
\left(\phi^{-1} \otimes \phi^{-1}\right) \Delta^{\prime} \phi\left(r \otimes r^{\prime}\right) & =\left(\phi^{-1} \otimes \phi^{-1}\right) \sum_{i} r\left(-t_{i}^{1}\right) r^{\prime} t_{i}^{2} \otimes \beta_{i} \\
& =\sum_{i, j, k} r \underbrace{e_{j}^{1} t_{i}^{1} r^{\prime} t_{i}^{2} e_{j}^{2}}_{\text {in } Z} \otimes_{Z} r_{j} \otimes_{R} \beta_{i}\left(e_{k}^{1}\right) e_{k}^{2} \otimes_{Z} r_{k} \\
& \cong \sum_{i, j, k} r \otimes_{Z} e_{j}^{1} t_{i}^{1} r^{\prime} t_{i}^{2} e_{j}^{2} r_{j} \beta_{i}\left(e_{k}^{1}\right) e_{k}^{2} \otimes_{Z} r_{k} \\
& =\sum_{i, k} r \otimes t_{i}^{1} r^{\prime} t_{i}^{2} \beta_{i}\left(e_{k}^{1}\right) e_{k}^{2} \otimes r_{k} \\
& =\sum_{k} r \otimes e_{k}^{1} r^{\prime} e_{k}^{2} \otimes r_{k} \\
& =\sum_{k} r \otimes 1 \otimes e_{k}^{1} r^{\prime} e_{k}^{2} r_{k}=r \otimes 1 \otimes r^{\prime} .
\end{aligned}
$$

The smash product decomposition of the endomorphism ring End $A_{B} \cong A \rtimes S$ discussed in Section 1 is now seen to recover the tensor algebra decomposition 9 . $2.6]$ :

$$
\text { End } A_{B} \cong A \otimes_{R} R \otimes_{Z} R^{\mathrm{op}} \cong A \otimes_{Z} R^{\mathrm{op}}
$$

as ring isomorphisms, an exercise to the interested reader. 
We note that the involutive antipode $\tau$ defined on $S$ is given by $(a \in A, \alpha \in S)$

$$
\tau(\alpha)(a)=\sum_{i} r_{i} a \alpha\left(e_{i}^{1}\right) e_{i}^{2},
$$

where $r_{i}, e_{i}$ is an H-separability system. The antipode $\tau$ does not appear to transfer to an antipode on its $R$-dual right bialgebroid $T$ via the Eqs. (9), (10) and (11) (cf. [19]). However, under the additional hypothesis that $R$ is Azumaya over the center $Z$ of $A .1$ the isomorphisms in Proposition 3.4 and Proposition 4.2(3) lead us to the following.

Theorem 5.2. If $A \mid B$ is $H$-separable with centralizer $R$ an Azumaya $Z$-algebra, then $T^{\mathrm{op}}$ is a Hopf algebroid over $R$.

Proof. We note that $\left(T^{\mathrm{op}}, R, \tilde{s}, \tilde{t}, \Delta^{\prime}, \varepsilon^{\prime}\right)$ is a left bialgebroid where the product on $T^{\mathrm{op}}$ is given by $t t^{\prime}=t^{1} t^{\prime 1} \otimes{t^{\prime}}^{2} t^{2}, \tilde{s}(r)=r \otimes 1, \tilde{t}(r)=1 \otimes r$, which together induce from the left the ordinary $R$-bimodule structure on $\left(A \otimes_{B} A\right)^{B}, \Delta^{\prime}$ given by Eq. (6i)) and $\varepsilon^{\prime}$ given by Eq. (7).

Since $R$ is an f.g. projective $Z$-algebra by Proposition 4.2, we have a left bialgebroid $\left(E:=\operatorname{End}_{Z} R, R, \lambda, \rho, \Delta, \varepsilon\right)$ as in Example 3.1 However, $R \otimes R^{\mathrm{op}} \cong E$ as bialgebroids by Proposition 3.4 whence $E$ is a Hopf algebroid over $R$ (with antipode induced by the twist on $R \otimes R^{\mathrm{op}}$ ). It suffices to show that the algebra isomorphism $\psi: T^{\mathrm{op}} \rightarrow E$ given by $t \mapsto\left(r \mapsto t^{1} r t^{2}\right)$ is a bialgebroid homomorphism (w.r.t. $\left.\operatorname{id}_{R}\right)$.

It is clear that $\psi \tilde{s}=\lambda, \psi \tilde{t}=\rho$, and $\varepsilon \psi=\varepsilon^{\prime}$. For the final computation, we note that $1 \otimes r_{i} \in R \otimes R, \eta_{i}: r \mapsto e_{i}^{1} r e_{i}^{2}$ is a right D2 quasibasis for $R \mid Z$ where $e_{i}, r_{i}$ is an H-separability system for $A \mid B$. Moreover, $e_{i} \in T, \rho_{r_{i}} \in S$ is a left D2 quasibasis for $A \mid B$ as noted in Section 4 Then by Eq. (4)

$$
\begin{aligned}
(\psi \otimes \psi) \Delta^{\prime}(t) & =(\psi \otimes \psi)\left(\sum_{i} e_{i} \otimes_{R}\left(\rho_{r_{i}}\left(t^{1}\right) \otimes_{B} t^{2}\right)\right) \\
& =\sum_{i} e_{i}^{1}(-) e_{i}^{2} \otimes t^{1} r_{i}(-) t^{2} \\
& =\Delta(\psi(t)) .
\end{aligned}
$$

If $Z$ is also a field coinciding with the center of $B$, then $T^{\mathrm{op}}$ possesses a weak Hopf $Z$-algebra structure [12, Prop. 9.4]. As a closing remark, it is not known as this is written if the bialgebroids $S$ or $T^{\mathrm{op}}$ associated to a Frobenius D2 extension are Hopf algebroids.

\section{REFERENCES}

[1] G. Böhm and K. Szlachányi, A coassociative $C^{*}$-quantum group with nonintegral dimensions, Lett. Math. Phys. 35 (1996), 437-456. MR 97k:46080

[2] T. Brzezinski and G. Militaru, Bialgebroids, $\times_{A}$-bialgeras and duality, J. Alg., to appear. ArXiv: QA/0012164.

[3] Y. Doi and M. Takeuchi, Hopf-Galois extensions of algebras, the Miyashita-Ulbrich action, and Azumaya algebras, J. Algebra 121 (1989), 488-516. MR 90b:16015

[4] P. Etingof and D. Nikshych, Dynamical quantum groups at roots of 1, Duke Math. J. 108 (2001), 135-168. MR 2002f:17019

[5] P. Etingof and A. Varchenko, Exchange dynamical quantum groups, Comm. Math. Phys. 205 (1999), 19-52. MR 2001g:17025

\footnotetext{
${ }^{1}$ It is interesting to note that these are the conditions on $A \mid B$ that ensure that End $A_{B} \mid \lambda(A)$ is $\mathrm{H}$-separable [20.
} 
[6] D. Evans and Y. Kawahigashi, Quantum Symmetries on Operator Algebras, Oxford Univ. Press, New York, 1998. MR 99m:46148

[7] K. Hirata, Some types of separable extensions of rings, Nagoya Math. J. 33 (1968), 107-115. MR 38:4524

[8] K. Hirata, Separable extensions and centralizers of rings, Nagoya Math. J. 35 (1969), 31-45. MR 39:5636

[9] L. Kadison, New examples of Frobenius extensions, University Lecture Series 14, Amer. Math. Soc., Providence, 1999. MR 2001j:16024

[10] L. Kadison and D. Nikshych, Hopf algebra actions on strongly separable extensions of depth two, Adv. in Math. 163 (2001), 258-286.

[11] L. Kadison and D. Nikshych, Frobenius extensions and weak Hopf algebras, J. Algebra 244 (2001), 312-342. MR 2002i:16052

[12] L. Kadison and K. Szlachanyi, Bialgebroid actions on depth two extensions and duality, Adv. in Math., to appear.

[13] H. Kreimer and M. Takeuchi, Hopf algebras and Galois extensions of an algebra, Indiana Univ. Math. J. 30 (1981), 675-692. MR 83h:16015

[14] J.-H. Lu, Hopf algebroids and quantum groupoids, Int. J. Math. 7 (1996), 47-70. MR 97a:16073

[15] D. Nikshych, A duality theorem for quantum groupoids, in "New Trends in Hopf Algebra Theory," Contemp. Math. 267 (2000), 237-243. MR 2002c: 16052

[16] D. Nikshych and L. Vainerman, A characterization of depth 2 subfactors of $\mathrm{II}_{1}$ factors, $J$. Func. Analysis 171 (2000), 278-307. MR 2000m:46129

[17] D. Nikshych and L. Vainerman, A Galois correspondence for actions of quantum groupoids on $\mathrm{II}_{1}$-factors, J. Func. Analysis, 178 (2000), 113-142. MR 2001j:46102

[18] T. Onodera, Some studies on projective Frobenius extensions, J. Fac. Sci. Hokkaido Univ. Ser. I, 18 (1964), 89-107. MR 30:4790

[19] P. Schauenburg, Duals and doubles of quantum groupoids $\left(\times_{R}\right.$-Hopf algebras), in: "New trends in Hopf algebra theory," A.M.S. Contemp. Math. 267 (2000), 273-299. MR 2001i:16073

[20] K. Sugano, Note on separability of endomorphism rings, J. Fac. Sci. Hokkaido Univ. 21 (1971), 196-208. MR 45:3465

[21] M.E. Sweedler, The predual theorem to the Jacobson-Bourbaki theorem, Trans. A.M.S. 213 (1975), 391-406. MR 52:8188

[22] K. Szlachányi, Finite quantum groupoids and inclusions of finite type, Fields Inst. Comm. 30, Amer. Math. Soc., Providence, RI, 2001, 393-407. MR 2002j:18007

Matematiska Institutionen, Göteborg University, S-412 96 Göteborg, Sweden

E-mail address: kadison@math.chalmers.se

Current address: Department of Mathematics and Statistics, University of New Hampshire, Durham, New Hamphsire 03824

E-mail address: kadison@math.unh.edu 